\title{
Characterizations and the Mechanism Underlying Osteogenic Activity of Peptides from Enzymatic Hydrolysates of Stichopus japonicus
}

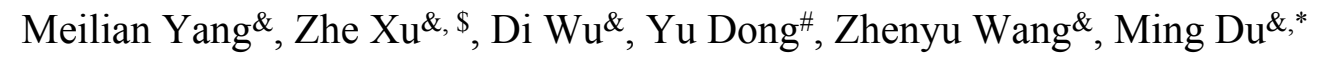

\& School of Food Science and Technology, Collaborative Innovation Center of Seafood Deep Processing, National Engineering Research Center of Seafood, Dalian Polytechnic University, Dalian 116034, China.

$\$$ College of Life Sciences, Key Laboratory of Biotechnology and Bioresources Utilization, Dalian Minzu University, Dalian 116029, China.

\# Dalian Feide Biological Industry Co., Ltd. Dalian 116085, China.

* Corresponding Authors: Prof. Ming Du (E-mail: duming@dlpu.edu.cn; Fax: +86-411-86323262; Tel: +86-411-86332275) 
Supplemental Table 1. Peptides from SJP Identified by LC-MS/MS.

\begin{tabular}{|c|c|c|c|c|c|c|c|}
\hline No. & AA sequence & $m / z$ & Mr.calc. & Protein & Score & $p I$ & $\begin{array}{l}\text { Hydrophilicity } \\
\text { (GRAVY) }\end{array}$ \\
\hline 1 & IHKGKVIRRVIVH & 519.0070 & 1557.0212 & Ig-like domain-containing protein (predicted) & 0.67 & 12.00 & 0.20 \\
\hline 2 & SVDYGKKSKLEFA & 736.3932 & 1472.7864 & Tubulin alpha chain & 124.60 & 8.20 & -0.72 \\
\hline 3 & KLVRRFWLRRSLKLKR & 431.8844 & 2159.4218 & $\begin{array}{l}\text { Putative DNA-directed RNA polymerase III subunit } \\
\qquad \text { RPC4-like(predicted) }\end{array}$ & 2.14 & 12.60 & -0.86 \\
\hline 4 & KVMKQVHPDTGIS & 720.3873 & 1440.7747 & Histone $\mathrm{H} 2 \mathrm{~B}$ & 144.17 & 8.60 & -0.52 \\
\hline 5 & FSLFDKDGDGTITTK & 822.9094 & 1645.8188 & Putative calmodulin isoform X1 (Fragment) & 168.31 & 4.43 & -0.54 \\
\hline 6 & GQMPSDKTLGGGDDSFNTF & 987.4309 & 1974.8618 & Tubulin alpha chain & 134.61 & 3.93 & -0.86 \\
\hline 7 & RRNLDIERPTYTN & 824.4317 & 1648.8634 & Tubulin alpha chain & 146.11 & 8.74 & -1.81 \\
\hline 8 & VREIAQDFKTELRFQ & 940.5049 & 1881.0097 & Histone $\mathrm{H} 3$, embryonic & 105.14 & 6.15 & -0.75 \\
\hline 9 & RDNIQGITKPAIR & 494.6231 & 1483.8692 & Histone H4 (Fragment) & 98.35 & 10.84 & -0.83 \\
\hline 10 & DEELNKLLSGVTIAQ & 815.4383 & 1630.8767 & Histone $\mathrm{H} 2 \mathrm{~A}$ & 144.10 & 4.14 & -0.09 \\
\hline 11 & RVTIMPKDIQLA & 692.9027 & 1385.8054 & Histone $\mathrm{H} 3$, embryonic & 114.89 & 8.75 & 0.25 \\
\hline 12 & IAQDFKTELRFQ & 748.3988 & 1496.7976 & Histone $\mathrm{H} 3$, embryonic & 219.72 & 6.07 & -0.62 \\
\hline 13 & AVGKVIPDLNGK & 605.8613 & 1211.7227 & Glyceraldehyde-3-phosphate dehydrogenase (Fragment) & 125.97 & 8.64 & 0.11 \\
\hline 14 & GSPIRIPVGPET & 611.8431 & 1223.6863 & ATP synthase subunit beta & 116.55 & 6.00 & -0.16 \\
\hline 15 & SRSARAGLQFPVG & 673.3704 & 1346.7408 & Histone $\mathrm{H} 2 \mathrm{~A}$ & 89.17 & 12.00 & -0.16 \\
\hline 16 & NDEELNKLLSGVTIAQ & 872.4598 & 1744.9196 & Histone $\mathrm{H} 2 \mathrm{~A}$ & 131.69 & 4.14 & -0.31 \\
\hline 17 & NDEELNKLLSGVT & 716.3699 & 1432.7398 & Histone $\mathrm{H} 2 \mathrm{~A}$ & 178.54 & 4.14 & -0.59 \\
\hline 18 & SSRAGLQFPVGRVH & 755.9155 & 1511.8310 & Histone $\mathrm{H} 2 \mathrm{~A}$ & 163.90 & 12.00 & -0.21 \\
\hline 19 & NADVNCVGKPYNL & 732.3510 & 1464.7020 & Orthopedia & 45.14 & 5.83 & -0.36 \\
\hline 20 & GQMPSDKTLGGGDDSFNTF & 987.4309 & 1974.8618 & Tubulin alpha chain & 134.61 & 3.93 & -0.86 \\
\hline 21 & EELNKLLSGVTIAQ & 757.9249 & 1515.8497 & Histone $\mathrm{H} 2 \mathrm{~A}$ & 104.22 & 4.53 & 0.15 \\
\hline 22 & AVLLRLPAVVVIL & 459.3182 & 1377.9545 & Transmembrane protein 19 & 1.64 & 9.79 & 2.62 \\
\hline
\end{tabular}




\begin{tabular}{|c|c|c|c|c|c|c|c|}
\hline 23 & KSYELPDGQVITIG & 760.4037 & 1520.8075 & Non-muscle actin II & 147.74 & 4.37 & -0.19 \\
\hline 24 & SRSARAGLQFPVGR & 501.2831 & 1503.8492 & Histone $\mathrm{H} 2 \mathrm{~A}$ & 148.70 & 12.30 & -0.47 \\
\hline 25 & DEELNKLLSGVT & 659.3485 & 1318.6969 & Histone $\mathrm{H} 2 \mathrm{~A}$ & 90.15 & 4.14 & -0.35 \\
\hline 26 & GINYQPPTVVPGGDLA & 799.4147 & 1598.8293 & Tubulin alpha chain & 106.40 & 3.80 & 0.00 \\
\hline 27 & QTNLVPYPRIH & 669.3698 & 1338.7397 & Tubulin alpha chain & 110.87 & 8.75 & -0.67 \\
\hline 28 & DLGLKIPGSDPLDM & 735.8791 & 1471.7581 & Putative filamin-B & 101.38 & 3.93 & -0.10 \\
\hline 29 & SARAGLQFPVGRVH & 747.9180 & 1495.8361 & Histone $\mathrm{H} 2 \mathrm{~A}$ & 134.38 & 12.00 & -0.02 \\
\hline 30 & GPQILNECGLQAKGSVNG & 921.4623 & 1842.9247 & CXC domain-containing protein(predicted) & 77.64 & 5.99 & -0.27 \\
\hline 31 & FVDLEPTVVDEVRTGTYR & 699.3601 & 2098.0804 & Tubulin alpha chain & 89.26 & 4.32 & -0.28 \\
\hline 32 & KTYELPDGQVITI & 738.9009 & 1477.8017 & Putative actin, cytoplasmic-like & 100.88 & 4.37 & -0.16 \\
\hline 33 & TSVLDPDEGIRFR & 502.2633 & 1506.7900 & Citrate synthase & 100.19 & 4.56 & -0.59 \\
\hline 34 & LIEDHFLFDKPVSP & 828.9352 & 1657.8705 & Putative creatine kinase, flagellar isoform X1 & 112.83 & 4.54 & 0.02 \\
\hline 35 & NDEELNKLLSGV & 665.8461 & 1331.6922 & Histone $\mathrm{H} 2 \mathrm{~A}$ & 219.35 & 4.14 & -0.58 \\
\hline 36 & FVDLEPTVVDEIRSG & 838.4305 & 1676.8610 & Tubulin alpha chain (Fragment) & 146.16 & 3.92 & 0.11 \\
\hline 37 & AIRNDEELNKLLS & 757.9123 & 1515.8246 & Histone $\mathrm{H} 2 \mathrm{~A}$ & 90.05 & 4.68 & -0.69 \\
\hline 38 & KQVHPDTGISSRAM & 509.5963 & 1528.7889 & Histone $\mathrm{H} 2 \mathrm{~B}$ & 81.90 & 8.75 & -0.75 \\
\hline 39 & EKNSTIIFPLPIE & 750.9190 & 1501.8381 & PHB domain-containing protein & 113.47 & 4.53 & 0.08 \\
\hline 40 & EYFRDQEGQDVLLF & 879.9203 & 1759.8406 & ATP synthase subunit beta & 136.96 & 3.92 & -0.70 \\
\hline 41 & PGELAKHAVSE & 569.2986 & 1138.5971 & Histone $\mathrm{H} 2 \mathrm{~B}$ & 106.40 & 5.41 & -0.48 \\
\hline 42 & VREIAQDFKTE & 668.3488 & 1336.6976 & Histone $\mathrm{H} 3$, embryonic & 103.31 & 4.68 & -0.89 \\
\hline 43 & AVGKVIPDLNGKLT & 712.9272 & 1425.8544 & Glyceraldehyde-3-phosphate dehydrogenase (Fragment) & 101.39 & 8.64 & 0.31 \\
\hline 44 & GFAGDDAPRAVFPS & 703.8386 & 1407.6772 & Non-muscle actin II & 84.29 & 4.21 & -0.08 \\
\hline 45 & ARAGLQFPVGRVH & 704.4020 & 1408.8041 & Histone $\mathrm{H} 2 \mathrm{~A}$ & 134.77 & 12.00 & 0.04 \\
\hline 46 & DFKTELRFQ & 1183.6106 & 1183.6106 & Histone $\mathrm{H} 3$, embryonic & 183.43 & 6.07 & -1.13 \\
\hline 47 & GPPGQAGLRGP & 1006.5429 & 1006.5429 & Alpha-2 collagen & 104.45 & 9.75 & -0.80 \\
\hline 48 & SSDALDKIRYE & 648.8251 & 1297.6502 & Heat shock protein 90 -alpha 3 & 132.32 & 4.56 & -1.06 \\
\hline
\end{tabular}




\begin{tabular}{|c|c|c|c|c|c|c|c|}
\hline 49 & DLSFIESNQFRLS & 778.3912 & 1556.7824 & $\begin{array}{l}\text { Putative choline O-acetyltransferase isoform X1 } \\
\text { (Fragment) }\end{array}$ & 94.69 & 4.37 & -0.25 \\
\hline 50 & IYEETRGVLKVF & 727.4061 & 1454.8122 & Histone H4 (Fragment) & 106.62 & 6.14 & 0.14 \\
\hline 51 & RRNLDIERPTYTNL & 880.9737 & 1761.9475 & Tubulin alpha chain & 128.88 & 8.74 & -1.41 \\
\hline 52 & KLTTPTYGDLNHL & 736.8908 & 1473.7816 & Tubulin beta chain & 137.01 & 6.74 & -0.62 \\
\hline 53 & ADQLTEEQIAEFK & 782.3805 & 1564.7610 & Putative calmodulin isoform X1 (Fragment) & 150.46 & 4.00 & -0.84 \\
\hline 54 & NDEELNKLLSGVTIA & 808.4305 & 1616.8610 & Histone $\mathrm{H} 2 \mathrm{~A}$ & 131.54 & 4.14 & -0.09 \\
\hline 55 & SVLDPDEGIRFR & 702.3675 & 1404.7350 & Citrate synthase & 112.30 & 4.56 & -0.58 \\
\hline 56 & IDDHFLFDKPVSP & 765.3854 & 1530.7707 & Creatine kinase, mitochondrial & 104.43 & 4.41 & -0.27 \\
\hline 57 & TIPEPNLPDGVSH & 688.3462 & 1376.6925 & Complex I-B14.5a & 123.86 & 4.35 & -0.61 \\
\hline 58 & RAGLQFPVGRVH & 668.8835 & 1337.7669 & Histone $\mathrm{H} 2 \mathrm{~A}$ & 200.88 & 12.00 & -0.11 \\
\hline 59 & IFRPDNFVFGQ & 670.3433 & 1340.6866 & Tubulin beta chain & 115.71 & 5.84 & 0.01 \\
\hline 60 & DKEGIPPDQQRLIF & 828.4412 & 1656.8824 & Polyubiquitin & 138.99 & 4.56 & -0.99 \\
\hline 61 & KNSTIIFPLPIEM & 759.9155 & 1519.8309 & PHB domain-containing protein & 111.61 & 6.00 & 0.49 \\
\hline 62 & VEKIPGESLFAPE & 708.3745 & 1416.7490 & $\begin{array}{l}\text { Putative muscle M-line assembly protein } \\
\text { unc- } 89 \text { (predicted) }\end{array}$ & 39.31 & 4.25 & -0.13 \\
\hline 63 & IGPPLLTPISED & 626.3452 & 1252.6904 & Putative radial spoke head protein 4-like A-like & 106.38 & 3.67 & 0.24 \\
\hline 64 & SARAGLQFPVGR & 420.2387 & 1260.7160 & Histone $\mathrm{H} 2 \mathrm{~A}$ & 101.35 & 12.00 & -0.11 \\
\hline 65 & IYEETRGVLK & 1207.6681 & 1207.6681 & Histone H4 (Fragment) & 214.96 & 6.14 & -0.53 \\
\hline 66 & DSGDGVSHTVPIYE & 738.3361 & 1476.6722 & Non-muscle actin II & 130.66 & 4.02 & -0.49 \\
\hline 67 & VLDSGDGVSHTVPIYEGY & 954.4547 & 1908.9094 & Non-muscle actin II & 134.13 & 4.02 & -0.03 \\
\hline 68 & KSYELPDGQVITIG & 760.4037 & 1520.8075 & Non-muscle actin II & 110.39 & 4.37 & -0.19 \\
\hline 69 & SARAGLQFPVG & 1102.6004 & 1102.6004 & Histone $\mathrm{H} 2 \mathrm{~A}$ & 105.52 & 9.47 & 0.29 \\
\hline 70 & LIDDHFLFDKPVSP & 821.9274 & 1643.8548 & Creatine kinase, mitochondrial & 96.76 & 4.41 & 0.02 \\
\hline 71 & AGLQFPVGRVH & 1180.6586 & 1180.6586 & Histone $\mathrm{H} 2 \mathrm{~A}$ & 138.54 & 9.80 & 0.29 \\
\hline 72 & LENVIRDAVTY & 646.8459 & 1293.6918 & Histone H4 (Fragment) & 158.47 & 4.37 & 0.14 \\
\hline
\end{tabular}




\begin{tabular}{|c|c|c|c|c|c|c|c|}
\hline 73 & ARAGLQFPVGR & 586.3384 & 1172.6767 & Histone $\mathrm{H} 2 \mathrm{~A}$ & 140.14 & 12.00 & -0.05 \\
\hline 74 & NSFVNDIFERIA & 712.8621 & 1425.7241 & Histone $\mathrm{H} 2 \mathrm{~B}$ & 123.63 & 4.37 & 0.11 \\
\hline 75 & GIGTVPVGRVE & 1083.6157 & 1083.6157 & Elongation factor 1-alpha (Fragment)(predicted) & 156.46 & 6.00 & 0.51 \\
\hline 76 & DLAGRDLTDYL & 626.3144 & 1252.6288 & Non-muscle actin II & 90.15 & 3.93 & -0.38 \\
\hline 77 & VVEPYNSILTTH & 686.8590 & 1373.7180 & Tubulin alpha chain & 113.66 & 5.24 & 0.12 \\
\hline 78 & LIDDHFLFDKPVS & 773.4010 & 1546.8020 & Creatine kinase, mitochondrial & 121.61 & 4.41 & 0.15 \\
\hline 79 & GPVEQLPDYNRIR & 778.9126 & 1557.8252 & Beta-MPP & 110.38 & 6.07 & -1.19 \\
\hline 80 & PDEIRDSLVDIR & 714.3781 & 1428.7562 & Major yolk protein 2 & 105.40 & 4.23 & -0.70 \\
\hline 81 & RIGAGAPVYLA & 1087.6259 & 1087.6259 & Histone $\mathrm{H} 2 \mathrm{~A}$ & 110.81 & 8.75 & 0.88 \\
\hline 82 & GVVILVLIGILLF & 456.9791 & 1370.9374 & Putative prominin-1-A & 1.64 & 5.52 & 3.33 \\
\hline 83 & AVDSLVPIGRGQ & 606.3408 & 1212.6815 & $\begin{array}{l}\text { Putative ATP synthase subunit alpha, mitochondrial } \\
\text { isoform X2 }\end{array}$ & 148.05 & 5.88 & 0.32 \\
\hline 84 & TLGGGDDSFNTF & 1230.5273 & 1230.5273 & Tubulin alpha chain & 90.60 & 3.56 & -0.38 \\
\hline 85 & AVNMVPFPRLH & 640.8503 & 1281.7005 & Tubulin beta chain & 143.85 & 9.80 & 0.39 \\
\hline 86 & QRIGAGAPVYL & 572.8273 & 1145.6546 & Histone $\mathrm{H} 2 \mathrm{~A}$ & 101.28 & 8.75 & 0.40 \\
\hline 87 & PQGPPGESIPGRPGLP & 778.4150 & 1556.8300 & Putative collagen alpha-1(XVIII) chain-like & 93.26 & 6.43 & -0.95 \\
\hline 88 & RIGAGAPVYL & 1016.5887 & 1016.5887 & Histone $\mathrm{H} 2 \mathrm{~A}$ & 173.38 & 8.75 & 0.79 \\
\hline 89 & DVTLPWTTQTPGS & 701.8461 & 1403.6922 & LRRCT domain-containing protein (predicted) & 101.11 & 3.80 & -0.55 \\
\hline 90 & GNAVFGNNALKW & 645.8331 & 1291.6662 & Alpha-amylase & 91.85 & 8.75 & -0.14 \\
\hline 91 & PTLQGPQPFVMPQ & 1439.7352 & 1439.7352 & Forkhead transcription factor P (Fragment) & 97.46 & 5.96 & -0.41 \\
\hline 92 & NSFVNDIFER & 1240.5957 & 1240.5957 & Histone $\mathrm{H} 2 \mathrm{~B}$ & 295.32 & 4.37 & -0.50 \\
\hline 93 & DLSFLAVSGPTWD & 704.3432 & 1408.6864 & Phospholipase B-like & 101.64 & 3.56 & 0.32 \\
\hline 94 & ELPDGQVITIGNE & 1384.6955 & 1384.6955 & Non-muscle actin II & 129.00 & 3.57 & -0.28 \\
\hline 95 & GWVTIEPKPGPF & 664.3559 & 1328.7118 & $\mathrm{C}$-CAP/cofactor C-like domain-containing protein & 105.78 & 6.00 & -0.26 \\
\hline 96 & LGGGDDSFNTF & 1129.4796 & 1129.4796 & Tubulin alpha chain & 116.25 & 3.56 & -0.35 \\
\hline 97 & QEASEAYLVGL & 1179.5892 & 1179.5892 & Histone $\mathrm{H} 3$, embryonic & 120.87 & 3.79 & 0.22 \\
\hline
\end{tabular}




\begin{tabular}{|c|c|c|c|c|c|c|c|}
\hline 98 & VGIGGDPFNGT & 1033.4949 & 1033.4949 & $\begin{array}{c}\text { Succinate--CoA ligase [ADP/GDP-forming] subunit } \\
\text { alpha, mitochondrial }\end{array}$ & 139.02 & 3.80 & 0.06 \\
\hline 99 & ELPDGQVITIG & 1141.6099 & 1141.6099 & Non-muscle actin II & 100.98 & 3.67 & 0.31 \\
\hline 100 & GVLNPGHPHIM & 594.3031 & 1188.6063 & Putative creatine kinase, flagellar isoform X1 & 103.02 & 6.92 & 0.05 \\
\hline 101 & GPAGPPGPNGI & 933.4789 & 933.4789 & Putative collagen alpha-1(I) chain & 158.12 & 5.52 & -0.47 \\
\hline 102 & SFVNDIFERIAAE & 755.8805 & 1511.7609 & Histone $\mathrm{H} 2 \mathrm{~B}$ & 83.40 & 4.14 & 0.24 \\
\hline 103 & VDWADPIIEPD & 1269.5998 & 1269.5998 & Heterogeneous nuclear ribonucleoprotein Q & 110.22 & 3.37 & -0.28 \\
\hline 104 & VNDIFERIAAE & 638.8302 & 1277.6605 & Histone $\mathrm{H} 2 \mathrm{~B}$ & 150.15 & 4.14 & 0.10 \\
\hline 105 & NLDIERPTYTNL & 724.8726 & 1449.7453 & Tubulin alpha chain & 101.38 & 4.37 & -0.89 \\
\hline 106 & PPTVVPGGDLAK & 575.8270 & 1151.6539 & Tubulin alpha chain & 133.23 & 6.26 & 0.03 \\
\hline 107 & GPPGESIPGRPGLP & 665.8593 & 1331.7187 & Putative collagen alpha-1(XVIII) chain-like & 95.50 & 6.00 & -0.72 \\
\hline 108 & AVLVDLEPGTM & 1160.5868 & 1160.5868 & Tubulin beta chain & 125.25 & 3.67 & 0.91 \\
\hline 109 & SKPVDPDDPEIPFTEE & 907.9202 & 1815.8403 & Putative adenylate kinase 7 & 101.89 & 3.71 & -1.33 \\
\hline 110 & GETGPIGPPGSP & 1065.5211 & 1065.5211 & Putative EMI domain-containing protein 1 & 98.03 & 4.00 & -0.71 \\
\hline 111 & GVTFPSPP & 801.4141 & 801.4141 & Extracellular matrix protein 3 & 178.24 & 5.52 & 0.04 \\
\hline 112 & ANPVEGDIGAVF & 1188.5895 & 1188.5895 & $\begin{array}{l}\text { Putative trifunctional enzyme subunit alpha, } \\
\text { mitochondrial }\end{array}$ & 150.53 & 3.67 & 0.53 \\
\hline 113 & IQDKEGIPPDQQRLIF & 949.0125 & 1898.0251 & Polyubiquitin & 155.66 & 4.56 & -0.81 \\
\hline
\end{tabular}

